\title{
Some Results on Commutativity of Rings
}

\author{
M. S. S. KHAN
}

Abstract. Some new results on commutativity theorems of torsion free unital rings have been obtained.

Throughout the paper, $R$ will denote an associative ring, and $Z(R)$ the center of $R$. As usual, for any $x, y, \in R$, the commutator $[x, y]=x y-y x$ and the anticommutator $x o y=x y+y x$.

A ring $R$ is said to be commutative or anticommutative according as $[x, y]=0$ or $x \circ y=0$, for all $x, y \in R$.

An element $x \in R$ is said to be m-torsion free if $m x=0$ implies $x=0$, where $m$ is a positive integer. It is logically interesting to investigate how far a ring is commutative or anticommutative if $[x y, y x]=0$ or $x y o y x=0$. Motivated by these observations, Gupta [2] proved that a division ring $R$ is commutative if and only if $[x y, y x]=0$. A number of authors $[2,3]$ have extended this result in several ways. Awtar [1] established that a semi prime ring $R$ in which $[x y, y x] \in Z(R)$ is necessarily commutative. In the same paper the possibility of extending the result for arbitrary rings has been ruled out in view of the readily available noncommutative ring of $3 \times 3$ strictly, upper triangular matrices over the ring $Z$ of integers which satisfies the above condition.

The following example shows that the above result is not valid for arbitrary rings even if it is unital.

Example 1. Let

$$
R=\left\{\left(\begin{array}{ccc}
a & b & c \\
0 & a & d \\
0 & 0 & a
\end{array}\right): a, b, c, d, \in Z\right\}
$$

Then $R$ is a non-commutative unital ring for which $[x y, y x] \in Z(R)$ for all $x, y$ in $R$.

Therefore, if one replaces $Z$ by $(G F(p))_{2}$ in the above example, then $R$ satisfies both the properties $[[x y, y x], x]=0$ and $[x y o y x, x]=0$, but $R$ is not commutative.

1991 Mathematics Subject Classification. Primary: 16U80; Secondary: 16 U90.

Key words and phrases. Commutativity of Rings, Anticommutator, Unital Ring, Torsion Free Ring. 
One can observe that the ring $(G F(p))_{2}$ in the above example is of characteristic 2 and some appropriate conditions on the characteristic of the ring implies commutativity.

In this note, we prove two new results on commutativity of unital rings.

Theorem 1. Let $R$ be an unital ring with $[x y o y x, x]=0$ for all $x, y \in R$. If $R$ is a 2-torsion free ring, then $R$ is commutative.

Proof. By the hypothesis, we have

$$
x\left(x y^{2} x+y x^{2} y\right)=\left(x y^{2} x+y x^{2} y\right) x, \quad \text { for all } \quad x, y \in R .
$$

So

$$
x\left[y^{2}, x\right] x+\left[y x^{2} y, x\right]=0 .
$$

Replacing $x$ by $x+1$ in (1), we get

$$
\left[y^{2}, x\right]+x\left[y^{2}, x\right]+\left[y^{2}, x\right] x+x\left[y^{2}, x\right] x+\left[y^{2}, x\right]+2[y x y, x]+\left[y^{2} x y, x\right]=0 .
$$

Using (1), equation (2) becomes

$$
x\left[y^{2}, x\right]+\left[y^{2}, x\right] x+2\left[y^{2}, x\right]+2[y x y, x]=0 .
$$

Now, replacing $x$ by $x+1$ in (3) and using (3), one gets $4\left[y^{2}, x\right]=0$.

Since $R$ is $2-$ torsion free, this gives

$$
\left[y^{2}, x\right]=0 .
$$

Finally, replacing $y$ by $y+1$ in (4) and using (4), we obtain $2[y, x]=0$.

This implies $[y, x]=0$ and yields the required result. This completes the proof.

Now, we shall prove the following result in a more general setting.

Theorem 2. Let $R$ be an unital ring for which $\left[x y^{m} x-y x^{m} y, x\right]=0$ for all $x, y$ in $R$. If $R$ is $m$ !-torsion-free ring, then $R$ is commutative.

Proof. By our assumptions, we have

$$
x\left(x y^{m} x-y x^{m} y\right)=\left(x y^{m} x-y x^{m} y\right) x \quad \forall x, y \in R .
$$

This implies that

$$
x\left[y^{m}, x\right] x=\left[y x^{m} y, x\right]
$$

Replacing $x$ by $x+1$ in (4), we get

$$
\begin{aligned}
& {\left[y^{m}, x\right]+x\left[y^{m}, x\right]+\left[y^{m}, x\right] x+x\left[y^{m}, x\right] x=} \\
& {\left[y\left(1+{ }^{m} C_{1} x+{ }^{m} C_{2} x^{2}+\cdots+{ }^{m} C_{m} x^{m}\right) y, x\right]}
\end{aligned}
$$

Using (4) in (6), we obtain

(7) $\left[y^{m}, x\right]+x\left[y^{m}, x\right]+\left[y^{m}, x\right] x=\left[y\left(1+{ }^{m} C_{1} x+{ }^{m} C_{2} x^{2}+\cdots+{ }^{m} C_{m-1} x^{m-1}\right) y, x\right]$ 
Replacing $x$ by $1+x$ in (7) and combining with the result thus obtained, we get

$$
\begin{gathered}
2\left[y^{m}, x\right]=\left[y \left\{m+{ }^{m} C_{2}\left(1+{ }^{2} C_{1} x\right)+{ }^{m} C_{3}\left(1+{ }^{3} C_{1} x+{ }^{3} C_{2} x^{2}\right)\right.\right. \\
+{ }^{m} C_{4}\left(1+{ }^{4} C_{1} x+{ }^{4} C_{2} x^{2}+4 C_{3} x^{3}\right) \cdots \\
\cdots+{ }^{m} C_{m-1}\left(1+{ }^{m-1} C_{1} x+{ }^{m-1} C_{2} x^{2}+\cdots\right. \\
\left.\left.\cdots+{ }^{m-1} C_{m-2} x^{m-2}\right)\right\} y, x
\end{gathered}
$$

Repeating the same arguments $3 r d$ and 4 th times, one gets

$$
\begin{aligned}
& 0=\left[y \left\{{ }^{m} C_{2}{ }^{2} C_{1}+{ }^{m} C_{3}\left({ }^{3} C_{1}+{ }^{3} C_{2}\left(1+{ }^{2} C_{1} x\right)\right)+\right.\right. \\
& +{ }^{m} C_{4}\left({ }^{4} C_{1}+{ }^{4} C_{2}\left(1+{ }^{2} C_{1} x\right)+{ }^{4} C_{3}\left(1+{ }^{3} C_{1} x+{ }^{3} C_{2} x^{2}\right)+\cdots+\right. \\
& +{ }^{m} C_{m-1}\left({ }^{m-1} C_{1}+{ }^{m-1} C_{2}\left(1+{ }^{2} C_{1} x\right)+{ }^{m-1} C_{3}\left(1+{ }^{3} C_{1} x+{ }^{3} C_{2} x^{2}\right)+\cdots+\right. \\
& \left.\left.\left.\left.+{ }^{m-1} C_{m-2}\left(1+{ }^{m-2} C_{1} x+{ }^{m-2} C_{2} x^{2}+\cdots+{ }^{m-2} C_{m-3} x^{m-3}\right)\right)\right)\right\} y, x\right]
\end{aligned}
$$

This gives

$$
\begin{aligned}
0= & {\left[y \left\{{ }^{m} C_{3}{ }^{3} C_{2}{ }^{2} C_{1}+{ }^{m} C_{4}\left({ }^{4} C_{2}{ }^{2} C_{1}+{ }^{4} C_{3}\left({ }^{3} C_{1}+{ }^{3} C_{2}\left(1+{ }^{2} C_{1} x\right)\right)\right)+\right.\right.} \\
+ & \cdots+{ }^{m} C_{m-1}\left({ }^{m-1} C_{2}{ }^{2} C_{1}+{ }^{m-1} C_{3}\left({ }^{3} C_{1}+{ }^{3} C_{2}\left(1+{ }^{2} C_{1} x\right)\right)+\cdots\right. \\
\cdots+ & \cdots+{ }^{m-1} C_{m-2}\left({ }^{m-2} C_{1}+{ }^{m-2} C_{2}\left(1+{ }^{2} C_{1} x\right)+\cdots\right. \\
& \cdots+{ }^{m-2} C_{m-3}\left(1+{ }^{m-3} C_{1} x+{ }^{m-3} C_{2} x^{2}+\right. \\
& \left.\left.\left.\left.\left.\cdots+{ }^{m-3} C_{m-4} x^{m-4}\right)\right)\right)\right\} y, x\right]
\end{aligned}
$$

Hence, repeating the process of replacing $x$ by $x+1 m$ times, and using the previously obtained results at each stage, equation (10) yields

$$
0=\left[y\left({ }^{m} C_{m-1}{ }^{m-1} C_{m-2}{ }^{m-2} C_{m-3} \cdots{ }^{2} C_{1}\right) y, x\right] .
$$

This implies that

$$
m !\left[y^{2}, x\right]=0
$$

Since $R$ is a $m$ ! torsion free ring, we obtain

$$
\left[y^{2}, x\right]=0 \text {. }
$$

Now as in the proof of theorem 1, equation (11) can be used to show that $R$ is commutative. This completes the proof. 


\section{REFERENCES}

[1] R. Awtar, A remark on the commutativity of certain rings, Proc. Amer. Math. Soc. 41 (1973) 370-372.

[2] R. N. Gupta, Nilpotent matrices with invertible transpose, Proc. Amer. Math. Soc. 24 (1970) 572-575.

[3] Y. Hirano and H. Tominaga, A commutativity theorem for semiprime rings, Math. Japan 25 (1980), 665-667.

Department of Mathematical Sciences Mathematics Building

The University of Montana

Missoula, MT 59812-0864

USA

E-mail address: shoeb21@hotmail.com 